\title{
High-Grade Transformation of Adenoid Cystic Carcinoma Delineated with a Fibrous Rim: A Case Report
}

\author{
Hamide Sayar ${ }^{1}$, Sülen Sarıoğlư${ }^{2}$ Sevgi Bakariş ${ }^{1}$, Illhami Yıldırım³ ${ }^{3}$ Hüseyin Öztarakçı ${ }^{4}$ \\ ${ }^{1}$ Department of Pathology, Kahramanmaraş Sütçü Imam University Faculty of Medicine, Kahramanmaraş, Turkey \\ ${ }^{2}$ Department of Pathology, Dokuz Eylül University Faculty of Medicine, Izmir, Turkey \\ ${ }^{3}$ Department of Otolaryngology, Kahramanmaraş Sütçü Imam University Faculty of Medicine, Kahramanmaraş, Turkey \\ ${ }^{4}$ Department of Otolaryngology, Necip Fazıl Şehir State Hospital, Kahramanmaraş, Turkey
}

\begin{abstract}
Background: High-grade transformation or dedifferentiation in carcinoma is progression of a low-grade malignant neoplasm to a high-grade carcinoma or poorly differentiated adenocarcinoma. This is rarely observed in adenoid cystic carcinoma of the salivary glands.

Case Report: A 39 year-old woman presented with a painless mass at the left submandibulary region that had been growing slowly for 5 years. Submandibulary mass resection revealed a mass with peripheral adenoid cystic carcinoma and a central high-grade tumor delineated with a fibrous rim, raising the possibility of a hybrid or composite carcinoma, requiring differential diagnosis depending upon morphology and immunohistochemistry findings. The final histopathological diagnosis was high-grade transformation of adenoid cystic carcinoma. After surgical therapy, the patient was irradiated to the neck and submandibulary region. No sign of tumor recurrence has been evident for 36 months.
\end{abstract}

Conclusion: This present case seems to be another rare case with high-grade transformation of adenoid cystic carcinoma and the fibrous rim may be a histopathological feature of such cases, which should be kept in mind.

Key Words: Adenoid cystic carcinoma, submandibulary gland, dedifferentiation, high-grade transformation.

Received: 27.06.2012 Accepted: 10.04.2013

\section{Introduction}

Adenoid cystic carcinoma (ACC) is a well-described malignant tumor that occurs mainly in the major and minor salivary glands, including the seromucinous glands of the upper respiratory tract $(1,2)$. This tumor comprises roughly $1 \%$ of all malignant tumors of the oral and maxillofacial region and represents approximately $22 \%$ of all salivary gland epithelial malignancies (1). ACC is a slow-growing tumor consisting of ductal and myoepithelial cell composition with three major growth patterns: tubular, cribriform, and solid (3). Although slow growing, this tumor presents with late recurrences, distant metastases many years after presentation, and ultimately, fatal outcome $(4,5)$.

High-grade transformation (HGT) or dedifferentiated ACC is a rare variant of ACC, and is defined as transformation of a low-grade malignant neoplasm into a high-grade carcinoma or poorly differentiated adenocarcinoma $(4,6)$. This transformation may be more aggressive than conventional solid ACC and has been associated with recurrences, early distant metastasis, and greater mortality (3). Therefore, not recognising areas of HGT within ACC may result in the mismanagement of the therapy of these patients (7).

This paper is a report of a different case of ACC of the submandibulary gland, with gradually histologic transformation from lower-grade ACC to high-grade adenocarcinoma. To differentiate between the low-grade ACC and the high- grade transformed component, immunohistochemical analysis was used.

\section{Case Report}

Informed consent was obtained from the patient. A 39 year-old woman presented with a painless mass in the left submandibulary region that had been growing slowly for five years. Otorhinolaryngological examination revealed a submandibulary non-tender mass without fixation measuring 3 $\mathrm{cm}$ in diameter. Magnetic resonance imaging revealed a 30 $\mathrm{mm}$ well delineated lesion of the left submandibulary gland (Figure 1). The patient underwent a submandibulary mass resection.

The excised tissue measured $70 \times 70 \times 24 \mathrm{~mm}$ in size. The tumor itself measured $40 \times 30 \times 20 \mathrm{~mm}$ and showed yellowish, soft, and partially necrotic areas on gross examination. The tumor had well delineated borders without any capsule. Salivary gland tissue was identifiable adjacent to the tumor. The whole excised tissue was fixed with formalin and embedded in paraffin. Then, immunohistochemistry was performed on the samples after light microscopic examination.

Microscopic examination of the tumor revealed two distinct carcinomatous components. One of them had features consistent with a low-grade ACC, and the other component had features of a high-grade adenocarcinoma. The highgrade adenocarcinoma comprised approximately $85 \%$ of the

Address for Correspondence: Dr. Hamide Sayar, Department of Pathology, Kahramanmaraş Sütçü Imam University Faculty of Medicine, Kahramanmaraş, Turkey. Phone: +90344221 1431 - Ext:377 e-mail: hamide1976@yahoo.com 
tumor mass. Although both the low-grade ACC and highgrade adenocarcinoma components were evidently separated with a fibrous band, some focal areas revealed the intermixing of both tumor components (Figure 2a).

The low-grade ACC component was characterized by biphasic tubular, cribriform structures containing abluminal myoepithelial cells and luminal epithelial cells within a myxoid and/ or hyaline matrix. On cytological examination, the tumor cells were small, hyperchromatic, angulated and monomorphic. When viewed under a microscope, rare mitoses were iden-

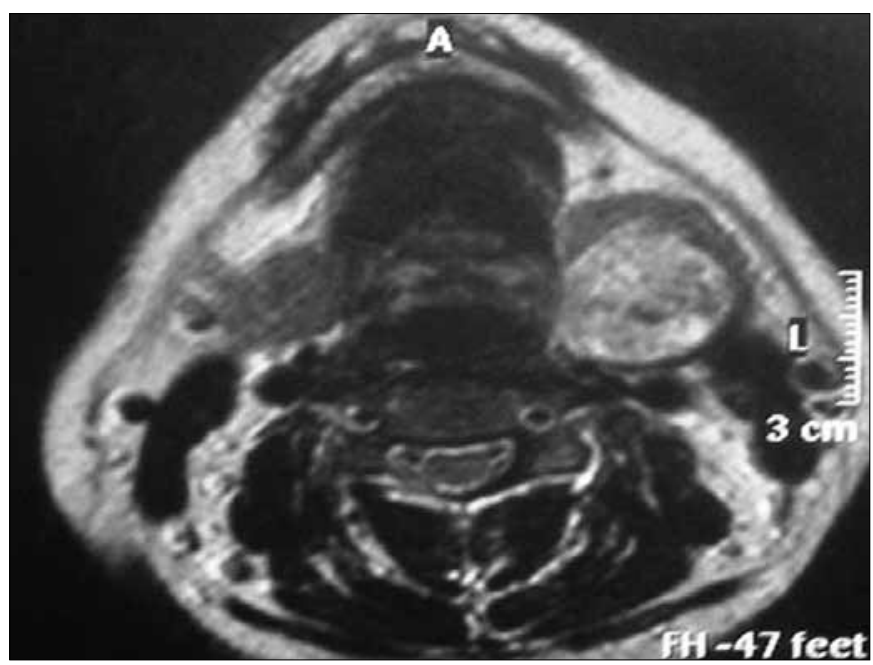

Figure 1. Magnetic resonance showing a lesion located in the left submandibulary gland.

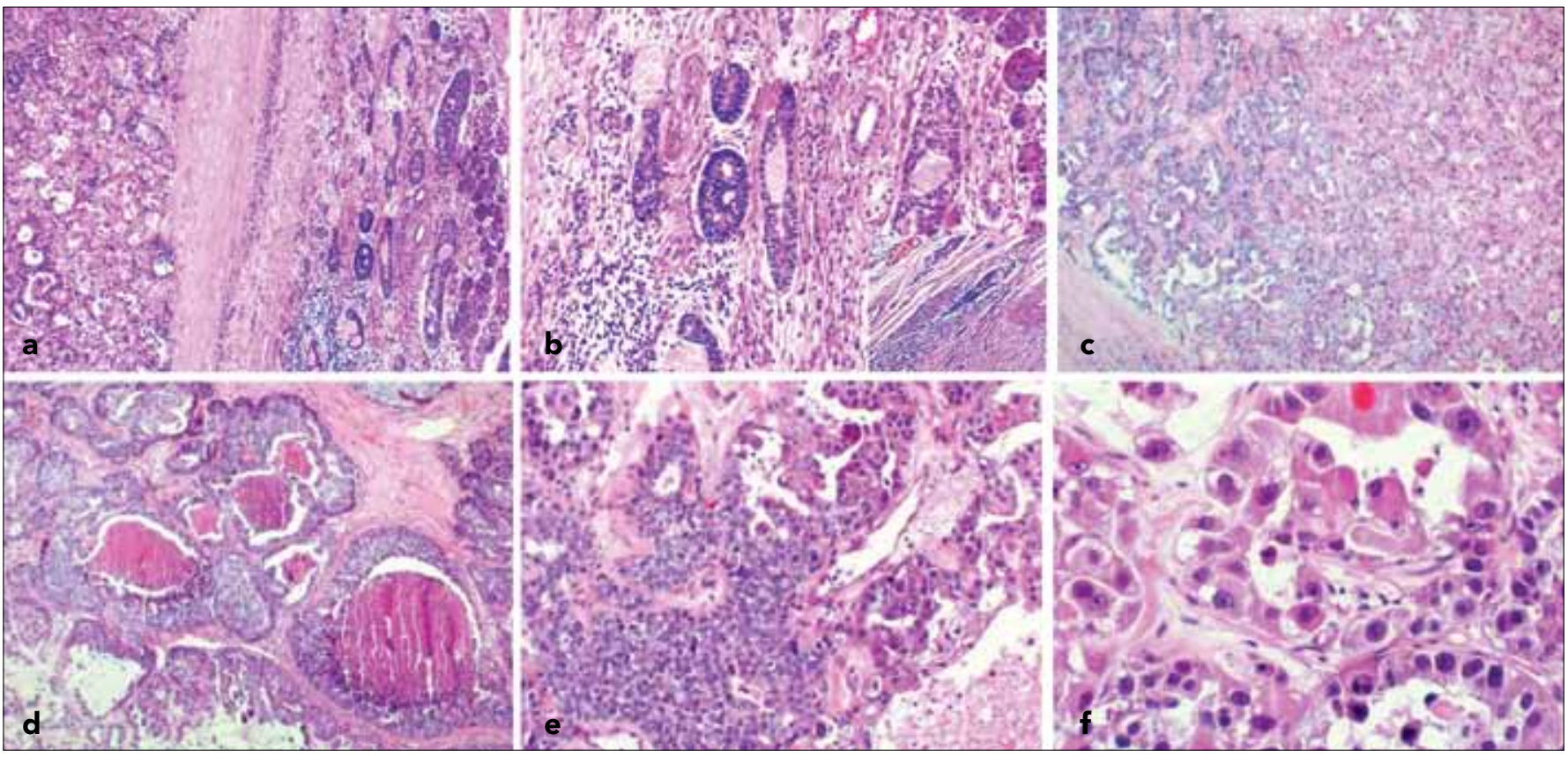

Figure 2. a-f. Microscopic findings (haematoxylin-eosin stain). Two distinct neoplastic cell populations separated by thin fibrous connective tissue are shown in the same tumor, on the left, low-grade adenoid cystic carcinoma and, on the right, HGT-ACC (magnification X40) (a). Low-grade ACC component showing a mixture of tubular, cribriform structures (magnification X 40) (b). Inset: note the perineural invasion of ACC (magnification X400). High-grade transformed component. Tubular growth pattern (magnification X40) (c). Micropapillary and solid growth patterns with comedo-like necrosis (magnification X40) (d). Transitional zone between solid adenoid cystic carcinoma and HGT-ACC (magnification X100) (e). Note the large polygonal cancer cells with conspicuous nucleoli (magnification X400) (f). tified; approximately one mitotic figure per ten high-power fields. The cyst-like spaces comprised of pale basophilic material as well as eosinophilic basal lamina material, which were positive for periodic acid-Schiff (PAS) stain. The ACC component also showed extensive perineural invasion (Figure 2b).

In comparison, the high-grade component showed glandular, solid, and sheet-like growth patterns with marked central necrosis, "comedonecrosis", made up of cells with large pleomorphic nuclei with conspicuous nucleoli, polygonal and large cytoplasms (Figure 2c-f) and a high mitotic rate $(50 \mathrm{mi}-$ totic figures per 10 high-power fields).

The immunohistochemical characteristics of the two tumor components are shown in Table 1. Immunohistochemically, the myoepithelial layer of the ACC was positive for SMA (Figures $3 a$ and $3 b$ ), Vimentin, and GFAP, whereas these expressions were lost in the transformed areas. CK5/6, CK7, 34 $\beta 12$, EMA and S100 (Figures $3 c$ and $3 d$ ) stained conventional ACC and HGT components with the same intensity, but CK20 was absent from both ACC and HGT components. The Ki-67 labelling index of ductal cells in HGT and epithelial cells of ACC was $70 \%$ and $20 \%$, respectively. Cyclin D1 overexpression was demonstrated in roughly $30 \%$ of the tumor cells in the HGT area, but it was only weakly expressed in the conventional ACC area. Staining for CD117 protein was positive in less than $20 \%$ of carcinoma cells both in the HGT and solid-type ACC areas, whereas it was negative in the lower-grade ACC portions. No staining for p53, CEA, or HER2/ neu was found in either the HGT or the ACC areas. The final pathological diagnosis was HGT- ACC of the submandibulary gland. 
Table 1. Comparison of immunohistochemical features in conventional ACC and high-grade components

\begin{tabular}{|lccc|}
\hline Antigens & Source & Conventional ACC & HGT \\
\hline CK 5/6 & Novacastro & + & + \\
CK7 & Leica & + & + \\
$34 \beta 12$ & NeoMarkers & + & + \\
CK20 & Leica & - & - \\
EMA & NeoMarkers & Luminal + & Luminal + \\
S100 & Leica & + & + \\
SMA & NeoMarkers & myoepithelial & + \\
GFAP & Cell Marque & myoepithelial & + \\
WIMENTIN & Leica & myoepithelial & + \\
GCDFP15 & Leica & - & - \\
CEA & Dako & - & - \\
P53 & Leica & - & - \\
CD117 (c-kit) & Leica & Weakly + & $20 \%+$ \\
CYCLIN D1 & Leica & - & $30 \%+$ \\
HER2/neu & Leica & - & - \\
Ki67 index & Leica & $20 \%$ & $70 \%$ \\
\hline
\end{tabular}

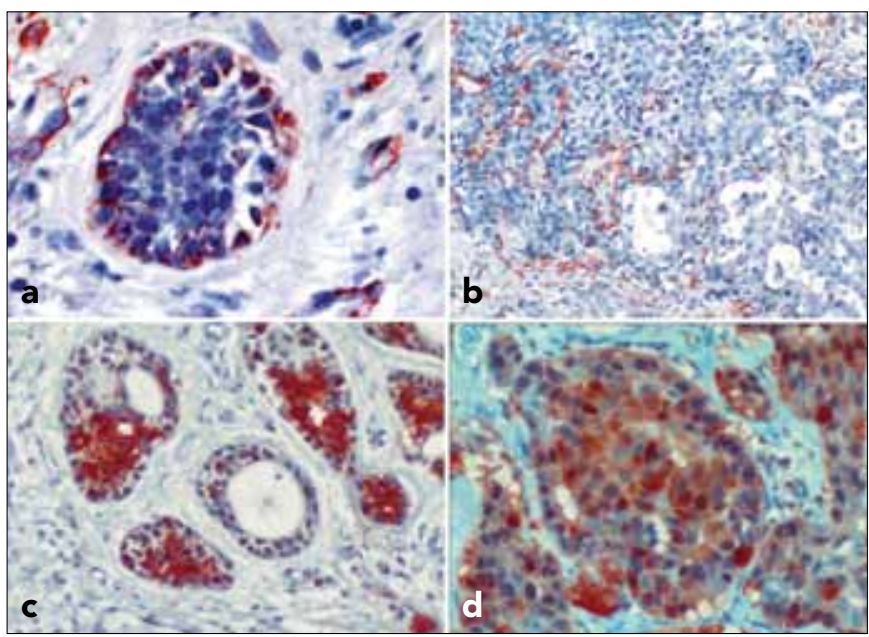

Figure 3. a-d. Immunohistochemical phenotypes of both components. Outer cells of ACC component was positive for SMA (magnification X200) (a). SMA, High-grade transition of ACC showing loss of myoepithelial layer (magnification X100) (b). S100, ACC area staining positive (magnification $\mathrm{X200}$ ) (c), S100, high-grade area staining positive (magnification X200) (d).

The patient underwent a second surgery for lymph node dissection. Lymph nodes obtained from the left functional neck dissection were negative for neoplasm. The histopathological surgical margin was tumor-free. The pathological stage was determined to be pT2N0Mx. After surgical therapy, the patient was irradiated to the neck and submandibulary region. No sign of tumor recurrence has been present for 36 months.

\section{Discussion}

HGT or dedifferentiation in carcinoma is the histological transformation of a low-grade malignant neoplasm to a highgrade carcinoma (3). Cheuk et al. (6) first reported a dedifferentiated variant of salivary ACC in 1999. Originally, dedifferentiation had been demonstrated in some mesenchymal tumors, such as dedifferentiated chondrosarcoma and dedifferentiated liposarcoma $(8,9)$. This statement has been adapted to salivary malignancies, such as ACC, acinic cell carcinoma, polymorphous low-grade adenocarcinoma, mucoepidermoid carcinoma, and myoepithelial carcinoma $(4,10)$. Some authors recommended the terminology of HGT rather than dedifferentiation (4); however, this is still controversial, because HGT is thought to reflect the actual tumor biology. In HGT-ACC, the high-grade tumor frequently preserves some features like glandular differentiation of the original tumor and is even then still recognisable as a carcinoma (4).

HGT-ACC occurs mainly in the submandibulary gland and the minor salivary glands in the sinonasal tract or palate (7). The age of HGT-ACC patients in a recent review was reported to range from 32 to 74 years $(n=24)$ with a mean age of 58 years. HGT-ACC shows a slight male predominance, whereas conventional ACC has a slight female predilection (4). For the present case, the patient's age and the site of the HGT-ACC were similar to previously reported cases.

HGT can be identified as an untreated, low-grade salivary gland tumor, in a recurrent lesion or sometimes after postoperative radiotherapy $(5,10,11)$. Our patient belongs to the former group. To our knowledge, there have been only 33 previously reported cases $(3,4)$. The amount of high-grade transformed component in ACC ranges from $10 \%$ to $80 \%$ $(4,10)$. In the present case, this component was found to be approximately $85 \%$ of the area. The high-grade transformed component was the predominant feature in most of the previous cases (10), as in our case.

Our case resembled the right maxillary sinus mass with central HGT described by Sato et al. (12), and the two cases of the submandibulary region presented by Chau at al. (10). With respect to cellular architecture, the tumor cells had small, hyperchromatic, angulated nucleus and were monomorphic in the conventional ACC component. The transformed component was defined as a pleomorphic high-grade proliferation of tumor cells with a common loss of myoepithelial cells around the tumor nests (5). In the present case, the fibrous rimming delineating two morphologically different components raised other possibilities. These entities include solid pattern ACC, hybrid carcinoma, and collision tumor (5). Discrimination of HGT-ACC from solid pattern ACC was occasionally difficult. Nuclear enlargement, chromatin irregularities, and comedonecrosis were more extensive in HGT-ACC than solid pattern ACC (4). In our case, the transformed component had prominent anisonucleosis and the formation of micropapilla, as described previously by Seethala et al. (4), as criteria for HGT, but lacked squamous metaplasia.

Hybrid carcinoma is a neoplasm composed of two or more disparate and well-established tumors showing obvious differentiation $(5,13)$. For our sample, some of the tumor nests of the HGT component with comedo-like necrosis, apocrine-like eosinophilic cytoplasm, coarse chromatin and distinct single 
nucleoli had findings suggestive of salivary duct carcinoma. These findings can be considered as adenoid cystic-salivary duct carcinoma hybrids. In addition to the loss of the myoepithelial layer and the presence of transition regions, immunoreactivity was not detected for CEA or GCDFP-15, which are frequently expressed in salivary duct carcinoma. Furthermore, even if there was loss of myoepithelial markers in the highgrade region, S100 was positive at both regions. Therefore, the HGT component was not assigned to a specific carcinoma entity and classified as an adenocarcinoma. Thus, the tumor in our case could be HGT-ACC rather than a hybrid carcinoma. Collision tumors are defined as a union of two separate neoplasms developing from different sites but close to each other (5). In our case, the presence of a transitional zone between the two carcinoma components and somehow similar immune profiles distinguished our tumor from a collision tumor.

The pathogenesis of HGT-ACC is not well understood. The HGT of malignant tumors are affected by the changes of various oncogenes and tumor suppressor genes. Recent studies have indicated that the abnormalities of p53, cyclin D1, and HER2/neu have been implicated in in the evolution of HGTACC $(4,5,10)$. In our study, immunohistochemical studies of p53, cyclin D1, CD117, and HER2/neu protein have been performed to explain the possible molecular mechanism. Cheuk et al. reported a role of the p53 gene and cyclin D1 in some cases of dedifferentiated ACC (6). In our case, the immunohistochemical negativity for p53 is considerable evidence to suggest that p53 may not be involved in the process of HGT. The cyclin D1 gene, with a central role in regulation of the G1 phase of the cell cycle, is frequently amplified in head and neck carcinomas. The HGT area in our sample exhibited positive staining for cyclin D1 protein, suggesting a possible role of cyclin D1 gene overexpression or amplification. Overexpression of CD117 has been found preferentially in high-grade areas of HGT-ACC. In our study, CD117 overexpression may have played a role in the dedifferentiation process of HGT-ACC. Previous studies reported variable results of HER2/neu in the high-grade component $(4,10,11)$. We found negative HER2/ neu in both components like the previous cases presented by Seethala and Chau $(4,10)$. Increased cell proliferative activity with (Ki-67 (MIB-1)) immunostaining in the transformed component of our case was also consistent with the high-grade malignant nature of this tumor.

HGT-ACC has an exceptionally poor prognosis with a median survival ranging from 12 to 36 months, while the survival time is approximately $36-48$ months in solid ACC $(3,4)$. Metastasis to the lymph nodes is frequent, as noted in $57 \%$ of the HGTACC patients versus $5-25 \%$ in conventional ACC patients (11). Because of the poor survival rate of metastasis, radical surgery along with radiotherapy is the best method of treatment (14). Considering the grave prognosis of the previous cases, the three year follow-up of the patient with no evidence of disease raises the possibility that a rim delineating the highgrade component may be a limiting and good prognostic factor in such patients.

In conclusion, this present case seems to be another rare case with ACC with HGT and the rim may be a histopathological feature of such cases, which should be kept in mind. HGT areas can be skipped if the samples obtained are not sufficient in number. To detect these transition zones and HGT areas, adequate samples should be investigated. Sometimes, if needed, the whole tumor mass should be sampled.

\section{Ethics Committee Approval: N/A}

Informed Consent: Written informed consent was obtained from the patient.

Peer-review: Externally peer-reviewed.

Author contributions: Concept - H.S., S.S.; Design - H.S., S.S., S.B.; Supervision - S.S., S.B., i.Y.; Resource - H.S., i.Y., H.Ö.; Materials - I.Y., H.Ö.; Data Collection\&/or Processing - H.S., H.Ö.; Analysis\&/or Interpretation - S.S., H.S., S.B.; Literature Search - H.S.; Writing - H.S., S.S.; Critical Reviews - H.S., S.S.

Conflict of Interest: No conflict of interest was declared by the authors.

Financial Disclosure: No financial disclosure was declared by the authors.

\section{References}

1. Jaso J, Malhotra R. Adenoid cystic carcinoma. Arch Pathol Lab Med 2011;135:511-5.

2. Malhotra KP, Agrawal V, Pandey R. High grade transformation in adenoid cystic carcinoma of the parotid: report of a case with cytologic, histologic and immunohistochemical study. Head Neck Pathol 2009;3:310-4. [CrossRef]

3. Costa $A F$, Altemani $A$, Vékony $H$, Bloemena $E$, Fresno $F$, Suárez $C$, et al. Genetic profile of adenoid cystic carcinomas (ACC) with high-grade transformation versus solid type. Cell Oncol (Dordr) 2011;34:369-79. [CrossRef]

4. Seethala RR, Hunt JL, Baloch ZW, Livolsi VA, Leon Barnes E. Adenoid cystic carcinoma with high-grade transformation: a report of 11 cases and review of the literature. Am J Surg Pathol 2007;31:1683-94. [CrossRef]

5. Nagao T, Gaffey TA, Serizawa H, Sugano I, Ishida Y, Yamazaki K, et al. Dedifferentiated adenoid cystic carcinoma: a clinicopathologic study of 6 cases. Mod Pathol 2003;16:1265-72. [CrossRef]

6. Cheuk W, Chan JK, Ngan RK. Dedifferentiation in adenoid cystic carcinoma of salivary gland: an uncommon complication associated with an accelerated clinical course. Am J Surg Pathol 1999;23:465-72. [CrossRef]

7. Seethala RR, Cieply K, Barnes EL, Dacic S. Progressive genetic alterations of adenoid cystic carcinoma with high-grade transformation. Arch Pathol Lab Med 2011;135:123-30.

8. Evans HL. Liposarcoma: a study of 55 cases with a reassessment of its classification. Am J Surg Pathol 1979;3:507-23. [CrossRef]

9. Dahlin DC, Beabout JW. Dedifferentiation of low-grade chondrosarcoma. Cancer 1971;28:461-6. [CrossRef]

10. Chau Y, Hongyo T, Aozasa K, Chan JK. Dedifferentiation of adenoid cystic carcinoma: report of a case implicating p53 gene mutation. Hum Pathol 2001;32:1403-7. [CrossRef]

11. Ide F, Mishima K, Saito I. Small foci of high-grade carcinoma cells in adenoid cystic carcinoma represent an incipient phase of dedifferentiation. Histopathology 2003;43:604-6. [CrossRef]

12. Sato K, Ueda $Y$, Sakurai A, Ishikawa $Y$, Kaji $S$, Nojima $T$, et al. Adenoid cystic carcinoma of the maxillary sinus with gradual histologic transformation to high-grade adenocarcinoma: a comparative report with dedifferentiated carcinoma. Virchows Arch 2006;448:204-8. [CrossRef]

13. Kamio N, Tanaka Y, Mukai M, Ikeda E, Kuramochi S, Fujii M, et al. A hybrid carcinoma: adenoid cystic carcinoma and salivary duct carcinoma of the salivary gland. An immunohistochemical study. Virchows Arch 1997;430:495-500. [CrossRef]

14. Seethala RR. Histologic grading and prognostic biomarkers in salivary gland carcinomas. Adv Anat Pathol 2011;18:29-45. [CrossRef] 